\title{
Traduire
}

Une autre perspective sur r tr traduction

Revue française de la traduction

$237 \mid 2017$

La tête dans la toile

\section{Traducteur en langue des signes-vidéo, un métier d'avenir}

Entretien avec Vivien Fontvieille

Morgane Boëdec, Vivien Fontvieille et Manon Rossetti

\section{(Q) OpenEdition \\ Journals}

Édition électronique

URL : http://journals.openedition.org/traduire/941

DOI : 10.4000/traduire.941

ISSN : 2272-9992

Éditeur

Société française des traducteurs

Édition imprimée

Date de publication : 1 décembre 2017

Pagination : 33-37

ISSN : 0395-773X

Référence électronique

Morgane Boëdec, Vivien Fontvieille et Manon Rossetti, « Traducteur en langue des signes-vidéo, un métier d'avenir », Traduire [En ligne], 237 | 2017, mis en ligne le 01 décembre 2017, consulté le 10 décembre 2020. URL : http://journals.openedition.org/traduire/941 ; DOI : https://doi.org/10.4000/ traduire.941 


\title{
Traducteur en langue des signes-vidéo, un métier d'avenir Entretien avec Vivien Fontvieille
}

\author{
Propos recueillis par Morgane Boëdec, \\ avec le concours de Manon Rossetti, interprète français-langue des signes (F-LSF).
}

Avant de commencer, l'interprète se présente et m'explique qu'elle va positionner la caméra de façon à ce que je ne voie que le traducteur, puis elle se place à côté de l'écran de l'ordinateur, face à lui. Pendant l'entretien, elle signe mes propos pour le traducteur et lui prête sa voix. Nous pouvons voir nos réactions mutuelles à l'écran, ce qui enrichit l'interprétation.

\section{Quel a été votre parcours pour devenir traducteur en langue des signes française (LSF) ?}

J'ai choisi, immédiatement après l'obtention du baccalauréat, de suivre une formation professionnelle à la traduction au CeTIM, le Centre européen de traduction, d'interprétation et de médiation de l'Université Toulouse-Jean-Jaurès.

L'ensemble des étudiants suivait les cours théoriques du tronc commun, et chacun rejoignait ensuite son groupe pour les modules linguistiques, qui en LSF, qui en allemand, en italien...

La spécificité de ce cursus en alternance sur trois ans est d'enseigner la théorie de la traduction tout en confrontant les étudiants de manière très régulière, en entreprise, aux réalités de la pratique de leur futur métier. C'est, à mon avis, une excellente formule. Je pense que l'exercice du métier est plus difficile pour ceux qui n'ont pas eu cette chance.

\section{Quelle place les nouvelles technologies ont-elles occupé dans votre formation ?}

Elles étaient très présentes : nous avions toujours recours au support vidéo, car nous traduisons en LSF. Alors que la traduction courante est toujours réalisée de l'écrit à l'écrit, en LSF, nous restituons nos traductions sous forme de vidéo, qui est le support écrit des Sourds(1). Le ministère de l'Éducation nationale l'a récemment reconnue comme tel, et les élèves scolarisés

(1) La majuscule à " Sourd " indique l'appartenance à la communauté Sourde signante, quand " sourd " fait référence à la déficience auditive. 
en LSF, par exemple, peuvent ainsi rendre leurs devoirs sous forme de vidéos. Je ne suis donc pas interprète, mais bien traducteur.

L'entretien que nous effectuons à l'instant est d'ailleurs possible grâce au recours à ces mêmes technologies!

\section{Quelle place occupent-elles dans votre travail aujourd'hui ?}

Internet est un formidable outil pour les traducteurs Sourds, travaillant en LS-vidéo, tout comme il l'est pour les traducteurs entendants travaillant avec l'anglais ou n'importe quelle autre langue : il permet d'avoir accès à des dictionnaires, des forums de discussion, de faire des recherches sur l'étymologie d'un mot ou d'un signe, etc.

Mais surtout, internet s'avère un formidable moyen de communication. Autrefois, les échanges ne se faisaient que par courrier postal, mais la messagerie électronique a considérablement élargi les possibilités de se contacter, d'échanger, de partager du contenu. Et alors que les vidéos ne se trouvaient auparavant qu'en DVD, les formats se sont désormais multipliés et il y a maintenant autant de supports possibles pour les LS-vidéo qu'il y a de sites internet. Accessibles depuis les quatre coins de la France (et au-delà évidemment !), les vidéos ont lissé les différences entre les nombreuses langues des signes régionales.

Internet s'est donc révélé un outil de travail très précieux pour nous et a, de plus, créé de nouveaux besoins pour la profession. La demande de traductions en LS-vidéo est maintenant bien plus importante, puisque les utilisateurs peuvent, depuis leur ordinateur, accéder à une énorme quantité d'informations, de ressources qu'ils souhaitent comprendre ; ce qui est désormais possible par le biais de vidéos, sans avoir à se déplacer pour en parler ni faire appel à des interprètes.

C'est donc bien grâce à l'arrivée d'internet que le métier a pu autant se développer et avec une telle rapidité.

\section{Vous dites que l'arrivée d'internet a lissé les différences régionales en LSF, pouvez- vous nous expliquer cela?}

Comme le français, la langue des signes française est empreinte de régionalismes à l'échelle du territoire. Les échanges ne se faisant auparavant que par le biais de rencontres physiques ou d'échanges de DVD, les influences d'une région sur une autre n'étaient pas très marquées.

Aujourd'hui, avec la plus grande accessibilité à ces vidéos, ces différences se sont lissées, ce qui peut provenir d'un souhait d'être compris de tous, voire d'une influence inconsciente, de l'adoption d'un signe que l'on rencontre plus souvent que celui utilisé localement pour la traduction d'un mot ou d'une idée. 


\section{Pensez-vous que ce soit une bonne chose ?}

II aurait été intéressant de garder ces différences régionales, car le risque de perte ou d'appauvrissement des langues locales existe. Mais je pense également qu'il est absolument essentiel que les informations soient diffusables au plus grand nombre : cela participe du droit à l'information - enfin accessible aux Sourds. Comme la vidéo le permet et le facilite, il faut continuer dans cette voie.

\section{À votre avis, la multiplication des vidéos traduites en LS contribue-t-elle à combler le retard dû aux cent ans d'interdiction de la LS en France(2) ?}

Oui, certainement, mais il n'est pas possible de comparer le développement des traductions en LS-vidéo avec celles en d'autres langues. Le retard est tellement important qu'il est loin d'être comblé, et le fait que le besoin même de traductions en LS-vidéo ne soit pas encore compris par tout le monde n'aide pas à mettre les bouchées doubles. Comme toutes les traductions, celles réalisées en LS-vidéo répondent à un besoin d'accessibilité, à un souci de diffusion de l'information et sont coûteuses. La volonté des donneurs d'ordre reste encore trop faible pour rattraper le retard.

\section{Revenons à votre métier : quelles sont vos combinaisons linguistiques de travail ?}

Je traduis à partir du français ou de l'anglais vers la langue des signes française (LSF) ou les signes internationaux (SI), via des vidéos (LS-vidéo). La LSF est ma langue maternelle, il est donc normal que ce soit ma langue d'arrivée.

Je travaille toujours à partir de supports écrits, puisque je suis traducteur, et il peut m'arriver de travailler depuis une vidéo en LSF, que je traduis sur un support vidéo en SI, ou l'inverse.

\section{Pouvez-vous nous expliquer comment vous organisez votre travail ?}

Ma méthode de travail consiste, après validation de la commande et réception du texte, en un premier temps de recherches : je le lis, m'informe du contexte et, en fonction de la provenance du texte, de son objet et de son auteur, je peux être amené à prendre contact avec le client pour m'assurer d'orienter ma traduction vers le bon public. Je relis à nouveau le texte et fais des recherches sur les signes propres au contexte ou à expliciter car peu usités.

(2) Proscrite au Congrès de Milan en 1880, la langue des signes n'est plus utilisée en France pendant un siècle qu'au sein des associations de Sourds. Dans les années 1980, au moment du "réveil sourd ", les Sourds réclament la réhabilitation de leur langue naturelle, son enseignement et sa reconnaissance officielle. 


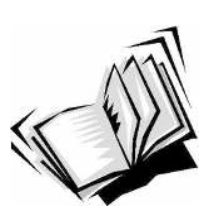

réception du texte

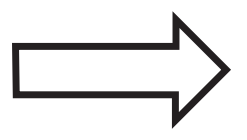

préparation de la traduction
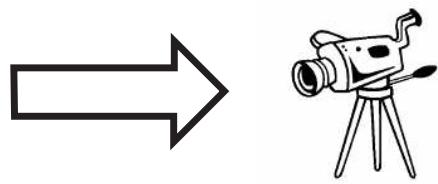

réalisation de la vidéo

Le travail de traduction commence alors par un schéma de déverbalisation de la langue française écrite et de reproduction en langue des signes vidéo. Je travaille l'adaptation culturelle et linguistique, puis mémorise le texte cible avant de passer à la troisième phase : celle du tournage de la traduction.

Je vérifie tous les aspects techniques (cadrage, etc.), puis je procède au tournage et à l'enregistrement. Je relis ensuite la vidéo, et réalise le montage du film que je livre alors au client.

Les traducteurs LS-vidéo expérimentés traduisent en moyenne 3000 mots par jour.

\section{Qui sont vos principaux clients?}

Les demandes me parviennent essentiellement de musées, pour les visioguides notamment, mais également de services publics, d'administrations et d'entreprises qui souhaitent rendre leurs informations accessibles en LSF.

\section{Avez-vous la possibilité de suivre des formations spécifiques tout au long de votre carrière ? Qui vous les propose et quelles en sont les modalités ?}

Oui, je continue à me former. J'ai choisi de m'intéresser à l'interprétation LSF-tactile(3), à destination des sourdaveugles. Je connais personnellement des sourdaveugles, ce qui m'a fait prendre conscience du réel manque d'interprètes LSF-tactile en France. Et je trouve important de continuer à acquérir des compétences, pour être polyvalent dans mon travail. Je suis encore jeune, j'en profite !

J'approfondis mes connaissances et ma pratique dès que j'en ai l'occasion, auprès des quelques centres qui proposent cette formation en France, et suis interprète LSF-Tactile depuis 2014, non seulement en France mais également en Europe.

(3) Les LS-tactiles permettent aux personnes sourdaveugles de communiquer, notamment en posant leurs mains sur celles de leur interlocuteur. 


\section{Vous arrive-t-il de créer du contenu, par le biais d'un blog par exemple, comme le font de nombreux traducteurs?}

Étant donné que mes traductions sont visuelles, et que l'on me connaît en tant que traducteur, la confusion serait possible si je créais du contenu vidéo ; je reste donc pour l'instant dans mon rôle de traducteur, et les vidéos sur lesquelles on peut me voir sont mes traductions, et non mes créations.

\section{Comment pensez-vous que votre profession évoluera à court et moyen termes ?}

J'espère que le nombre de formations à ce métier va augmenter; nous sommes certes suffisamment nombreux aujourd'hui pour répondre à la demande, mais cela ne va pas durer. Malheureusement, peu d'étudiants suivent la formation proposée à l'Université de Toulouse, car elle nécessite d'avoir à la fois un très bon niveau de LSF, de culture générale et un bon niveau de français lu et écrit.

Je suis également formateur au sein de ce cursus depuis 2012, et je pense que les besoins, enfin reconnus par la loi de 2005(4), feront exploser la demande, et que l'offre suivra. Je crois qu'une autre institution va prochainement proposer cette même formation dans le Nord de la France, car tout le monde ne peut aller à Toulouse pour se former.

\section{Y a-t-il un élément que vous souhaiteriez voir prendre plus de place ?}

Je voudrais vraiment que la traduction en LSF ne soit pas considérée comme moins importante, voire inférieure aux autres langues. Notre société doit évoluer et la langue des signes doit être reconnue à l'égale des autres.

traduquales@icloud.com

Vivien Fontvieille est traducteur français-LS vidéo depuis neuf ans. II travaille en tant que salarié au sein de la SCOP TIC44, basée à Nantes, et en tant que traducteur libéral sur le reste de la France.

Manon Rossetti est interprète français-LSF depuis six ans. Elle est co-fondatrice, associée et salariée de la SCOP TIC44 qui a trois ans maintenant. Elle en est co-gérante depuis un an.

(4) Loi 2005-102 du 11 février 2005 qui reconnaît la LSF comme une " langue à part entière ". 